\title{
Self-organized criticality of traffic flow
}

\author{
Jorge A. Laval ${ }^{1, *}$ \\ ${ }^{1}$ School of Civil and Environmental Engineering, Georgia Institute of Technology, \\ 790 Atlantic $\operatorname{Dr} N W$, Atlanta, GA, 30313, Atlanta, United States
}

(Dated: Version August 3, 2021)

\begin{abstract}
This paper shows that the kinematic wave model exhibits self-organized criticality when initialized with random initial conditions around the critical density. This has several important implications for traffic flow in the capacity state, such as: (i) jam sizes obey a power law distribution with exponents $1 / 2$, implying that both the mean and variance diverge to infinity, (ii) self-organization is an intrinsic property of traffic flow models in general, independently of other random perturbations, (iii) this critical behavior is a consequence of the flow maximization objective of traffic flow models, which can be observed on a density range around the critical density that depends on the length of the segment, (iv) typical measures of performance are proportional to the area under a Brownian excursion, and therefore are given by different scalings of the Airy distribution, (v) traffic in the time-space diagram forms self-affine fractals where the basic unit is a triangle, in the shape of the fundamental diagram, containing 3 traffic states: voids, capacity and jams.
\end{abstract}

Keywords: Traffic flow, kinematic wave model, self-organized criticality, fractals

\section{INTRODUCTION}

There is mounting empirical evidence [30-32] suggesting the hypotheses that urban networks exhibit selforganized criticality (SOC) [3], a celebrated paradigm in the 90's that has proved very useful in countless areas of science, from earthquakes to stock prices to forest fires to astrophysics $[2,6]$. The defining characteristic of a SOC system is that it is naturally driven to be in a "critical state" where it undergoes a phase transition [8] (e.g. from liquid to solid or from free-flow to congestion). At the critical point the characteristic length scale of the system, such as the size $S$ of forest fires, diverges to infinity, which renders the system scale-free and thus scale invariant. This implies a power law distribution for the size, which is proportional to $s^{-\alpha}$ :

$$
P(S>s) \sim s^{-\alpha},
$$

with the exponent $\alpha$ known as the critical exponent. As opposed to normal distributions, where it is virtually impossible to find realizations more than 3 standard deviations away from their mean value, power laws are said to have heavy tails: there is a large probability of observing extremely large values. As a consequence, the variance may diverge, in which case the central limit theorem no longer holds. When $\alpha>2$, both the mean and variance are finite; when $1<\alpha \leq 2$, as it is typically the case, the mean is finite and the variance diverges to infinity, and for $\alpha \leq 1$ both the mean and variance diverge. The case $\alpha=1$ is popularly known as Zipf's law [33], and as it turns out $\alpha \approx 1 \pm 0.2$ for a wide variety of phenomena, including the size of cities [18] and companies [10].

If it turns out that urban networks do exhibit SOC, a whole new paradigm emerges for understanding urban

\footnotetext{
* jorge.laval@ce.gatech.edu
}

congestion, based on the lessons learned from complexity, catastrophe theory and non-equilibrium critical phenomena. It would mean for example that most of our current control methods based on standard calculus and statistics may no longer apply or may need a major overhaul in existing methodologies. But given the absence of a general theory of SOC to date, it is not yet possible to formally prove or disprove this hypothesis.

The aim of this paper is to analyze the SOC behavior of the fundamental unit of urban networks: a single-lane uninterrupted road segment, where the only ingredients are the traffic flow model and the initial conditions. A solid understanding of this fundamental system is important to understand its consequences at the network level.

SOC behavior in traffic flow models has been conjectured since the mid-nineties in the physics community $[20,23]$. Let us define a jam as a congestion cluster in the time-space $(t, x)$ plane, i.e. a $(t, x)$-region of connected occupied "cells" representing stopped vehicles. Using ideas from the theory of percolation phase transitions they show that (i) the critical exponent of jam durations is very close to $1 / 2$, strongly suggesting a relationship with the first return time of a one-dimensional random walk, and (ii) the number of vehicles under congestion scales with time as $t^{1 / 2}$. They conclude that SOC implies that conventional traffic management strategies seeking to maximize the flow may be detrimental as they make the system more unpredictable and hard to control.

More recently, some contradictory evidence has been reported on critical behavior in freeway segments. [12] found that the flow time series on German freeways are well described by a fractional Brownian motion with Hurst exponent $H \approx 0.1$, which corresponds to a subdiffusive process with anti-correlated increments. In contrast, the same year [4] reports $H \approx 0.8$ for flows and speed on Australian motorways, which corresponds to a super-diffusive behaviour and long-range dependencies. Clearly, this large discrepancy cannot be explained by 
the location of the freeways, and more scrutiny is necessary.

But the impact of SOC in our field has been underwhelming [19, 26]. Apart from [21, 22, 25] who present some numerical evidence of SOC on networks, it appears that the subject has gone largely under the radar. One reason might be that the main insight from the early works was that SOC in single-lane models emerges only in "cruise-control" type traffic flow models, which only allow random perturbations in congested traffic states. This apparent dependence on the rule details might have been interpreted as the absence of a universality class in traffic flow. This paper presents evidence to the contrary.

\section{UNIVERSALITY OF TRAFFIC}

Possibly the most remarkable property of critical phenomena is universality, stating that critical exponents are determined by the dimensionality of the embedding space and the degrees of freedom of the variables in the system, and not by the details of the rules governing it.

A strong indication of universality for traffic flow is due to a symmetry of conservation laws pointed out in [14] whereby the specific shape of the fundamental diagram becomes irrelevant for the calculation of flows and delays.

The argument goes as follows. At the link level, it is widely accepted that traffic dynamics obey the kinematic wave model of $[16,24]$, which boils down to the conservation law PDE coupled with the existence of a fundamental diagram that gives the local flow as a function of the local density in the segment. The kinematic wave model is arguably the simplest model able to capture the transitions between free-flowing and congested conditions on a road segment each time the density in that segments exceeds the critical density.

Exploiting a symmetry in the kinematic wave model with respect to linear transformations of time and space, [14] showed that the numerical values for these parameters are irrelevant when it comes to the calculation of normalized flows and/or delays. This means that, effectively, traffic flow on a single link requires no parameters (after proper normalization) and therefore is independent of the details of the rules.

But a universality class also requires critical behavior. As seen in the introduction, [20, 23] observed this behavior for a very particular traffic flow model, which in fact corresponds to a non-deterministic variant of CA 184, where drivers slow down with a prescribed probability. Universality was not mentioned nor conjectured possibly because critical behavior was observed only for one specific rule, i.e. only when precluding freely-flowing drivers to randomly slow down.

Notably, the same invariance with respect to the fundamental diagram guarantees the equivalence between elementary cellular automata (CA) rule 184 [28] and the kinematic wave model with triangular fundamental. In fact, CA 184 is the simplest exact numerical solution

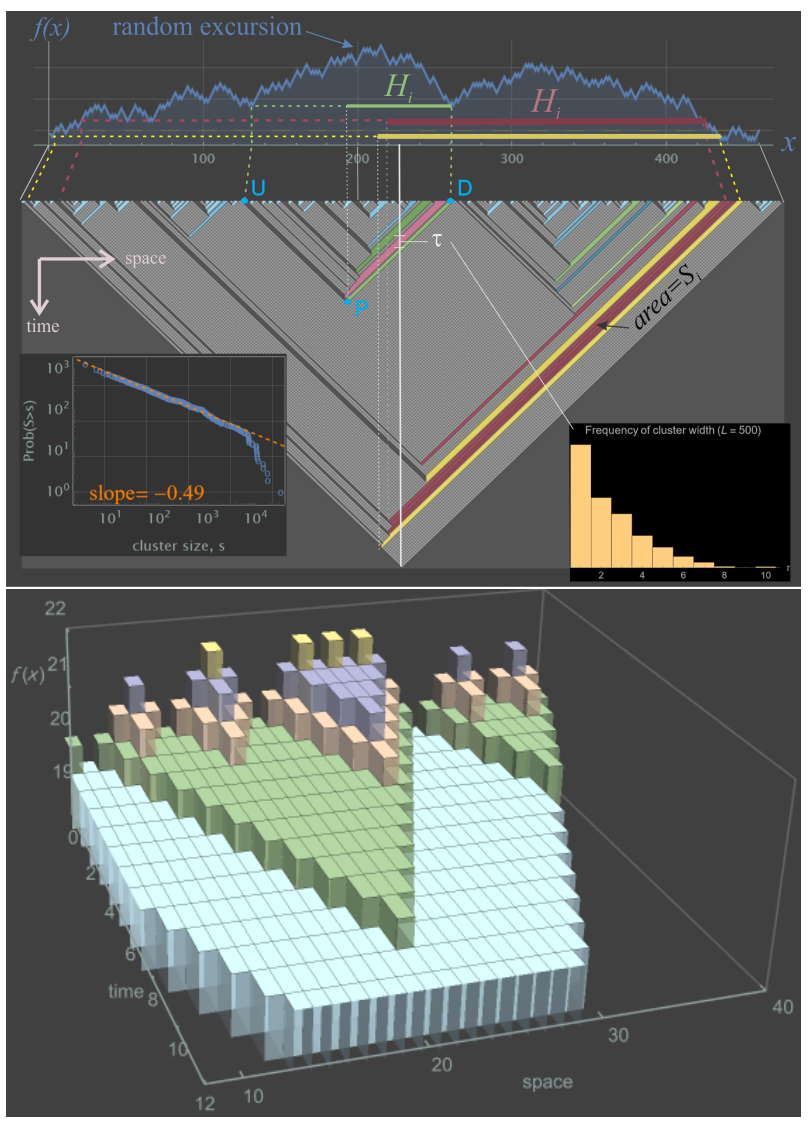

Figure 1. Correspondence between the area under the random walk and the space-time diagram. Top: Sample spatiotemporal realization for $\mathrm{CA}$ rule 184 under critical random initial conditions along with the cost function $f(x)$ which is a standard random walk. The colored bars represent the congestion clusters, the dark gray bars the voids and the light gray areas the capacity state. Bottom: a 3D representation of a zoomedin view of another realization. The third axis corresponds to deviations around the mean $\Delta N=N(P)-\mathrm{E}\{N(P)\}$, with $\mathrm{E}\{N(P)\}=(L+t-x) / 2$. The random walk can be seen as a slice of the surface at $t=0$, and the area under the random walk as the projection of the surface into the $(\Delta N, x)$-plane; note that state $\mathrm{C}$ is lost in this projection.

method of the kinematic wave model. In the next section, we use this solution method to study the system under random initial critical This isconditions.

\section{CRITICALITY OF CA RULE 184}

It was shown in [13] that the structure of congestion clusters in CA-184 exhibits power law behavior with an exponent close to $1 / 2$ at the critical density. This implies that both the mean and variance of the size of congested areas diverge, and cannot be tackled with traditional statistical methods. This fundamental feature traffic flow, which appears to have been overlooked in the literature so far, has important implications, as shown next. 


\section{A. Connection with random walks}

Here we show that at the critical density, traffic on a long single-lane road is fully characterized by a standard random walk. To show this, we exploit the fact that the kinematic wave model can be solved using the Hamilton-Jacoby PDE [5, 15], where the solution to the initial value problem with data $G(x)$ is given by the following variational problem, which in the case of CA 184 reads:

$$
N(P)=\min _{x_{U} \leq y \leq x_{D}} f(y) \equiv G(y)+(t-x+y) / 2,
$$

subject to initial conditions $N(0, x)=G(x)$ and where $x_{U}=x-t, x_{D}=x+t$ are the spatial coordinates of points $\mathrm{U}$ and $\mathrm{D}$ in Fig. 1 (top), $P \equiv(t, x)$ is a generic point in time-space, $N(P)$ is the cumulative number of vehicles that have crossed location $x$ by time $t$. Notice that all variables involved here are integers, as is customary for CA models. In particular, we consider here a road segment divided into $L$ cells, each with probability $\bar{k}$ of being occupied at $t=0$; i.e., the initial density $k_{i}^{0}, i=1,2, \ldots L$, can be either 0 or 1 with probabilities $1-\bar{k}$ and $\bar{k}$, respectively.

$$
G(x)=\sum_{i=x}^{L} k_{i}^{0}, \quad 1 \leq x \leq L
$$

The crucial point here is that at the critical density, the function to be minimized, $f(y)$, becomes constant and equal to $L / 2$ when initial conditions are deterministic. But more importantly, with random initial conditions this function is identical to a standard random walk. To see this, note that in terms of the initial densities the cost function can be written as:

$$
f(y)=\sum_{i=y}^{x+t}\left(k_{i}^{0}-1 / 2\right)+c,
$$

whose expected value is the $y$-independent term $c=$ $\sum_{i=x+t+1}^{L} k_{i}^{0}$ at the critical density since $\mathrm{E}\left\{k_{i}^{0}\right\}=\bar{k}=$ $1 / 2$. Since $\left(k_{i}^{0}-1 / 2\right)$ equals $1 / 2$ or $-1 / 2$ with equal probability, it follows that the random variable $f(y)-c$ is a random walk of increment $1 / 2$ at the critical density.

\section{B. Elementary jams}

Let us define an elementary jam as the collection of occupied cells connecting a point $\mathrm{P}$ located on the back of the queue and its corresponding downstream point $\mathrm{D}$ on the boundary; see Fig. 1. This is akin to a congested characteristic line connecting $\mathrm{P}$ to $\mathrm{D}$ but in discrete units. Points $\mathrm{P}$ in the back of the queue are characterized by a transition from free-flow to congestion at that point, which means that the cost of minimum paths P-U and PD both solve (2) and therefore have identical cost. This means that the duration $D-U$ in Fig. 1 is given by the first return time of our random walk $f(y)$, which are known to have a power law distribution with exponent $1 / 2$ [see e.g. 11, 27, p. 152]. From Fig. 1 it is clear that the duration, $H$, of an elementary jam is $H=(D-U) / 2$ and therefore retains the same power law distribution with exponent $1 / 2$ :

$$
P\left(H_{i}>h\right) \sim h^{-1 / 2}
$$

The consequences of this are far-reaching: both the mean and variance of elementary jam durations diverge to infinity, which renders standard statistical tools such as the central limit theorem inadequate for analyzing critical traffic flow.

\section{Cluster size distribution}

As mentioned to the introduction, [13] recently showed numerically that CA 184 exhibits power law behavior at the critical density, with cluster sizes, $S$, following a power law distribution with an exponent close to one half:

$$
P(S>s) \sim s^{-\alpha}, \quad \alpha \approx 1 / 2
$$

In terms of elementary jams, a jam can be characterized by a collection of, say $n$, contiguous elementary jams. The size $S$ of a jam can be seen as the sum of $n$ correlated power laws with exponents $1 / 2$ :

$$
S=\sum_{i=1}^{n} H_{i}
$$

It is well known that for independent $H_{i}$ 's and for large values of $S$ the power law approximation for $S$ is accurate [29], and maintains the same exponent, $1 / 2$ in this case, regardless of the value of $n$. In light of (6) one concludes that the correlations among the $H_{i}$ 's are not strong enough to alter the scaling exponent of cluster sizes.

Notice that the duration of consecutive congested states, $\tau$ in Fig. 1, is a measure of the width of the congested clusters, does not have a heavy-tail distribution, as shown in the figure. Recall that due to the symmetry property of the cumulative weight model, this is also the distribution of the duration of voids in a given location.

\section{Local flow distribution}

Let $q(t)$ be the flow crossing a stationary detector located in the middle of the segment at $x=L / 2$, as a function of time, and let $N(t)=\int_{0}^{t} q(s) d s$ be the corresponding cumulative count.

Fig. 2 shows the (oblique) cumulative counts and flow time series at 3 different locations for a typical rollout. 

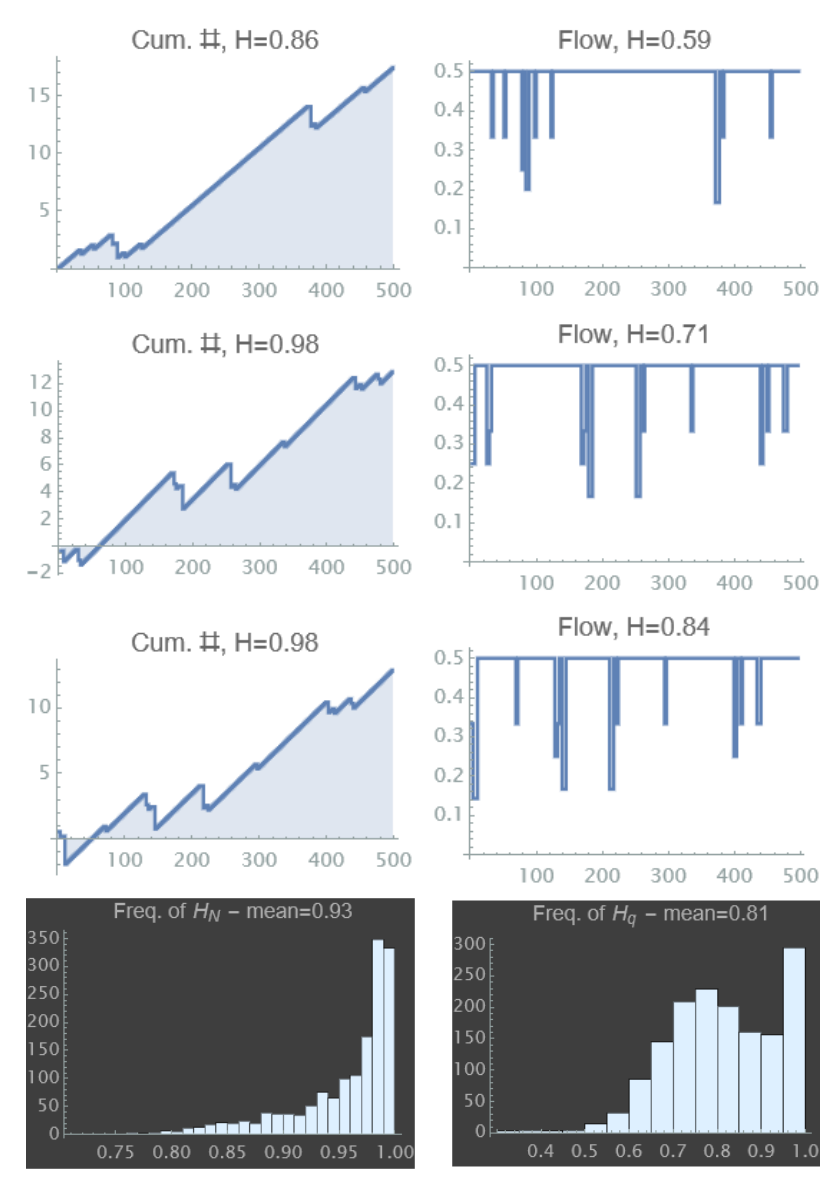

Figure 2. Oblique cumulative count $N(t)=\int_{0}^{t}(q(s)-0.45) d s$ and flow time series at 3 different locations for a typical rollout with $L=1000$. Each panel shows the Hurst exponent estimated by fitting a fractional Brownian motion process for the cumulative counts and a fractional Gaussian noise process for the flows. The histograms summarizes the results for 2000 rollouts.

The roughness of the cumulative curve is apparent as a result of the "spiky" variations in the flow around the capacity value $1 / 2$. Each panel shows the Hurst exponent estimated by fitting a fractional Brownian motion process for the cumulative counts and a fractional Gaussian noise process for the flows. The histograms summarizes the results for 2000 rollouts. The average exponent for the flows $H=0.81$ is in line with the empirical results reported for Australian motorways during morning rush hour and nights.

These high exponents indicate a long memory in the time series as a result of long-range correlations. In this case these correlations are due to the spatial interactions brought about by the traffic model, which imposes long periods of constant capacity flow interrupted by bursts of voids. Notice that the fractal dimension of the flow time series can be approximated by $D=2-H=2-0.81 \approx$ 1.2 .

\section{E. Aggregate measures of performance}

Let $\Psi \equiv \int_{A} k(t, x) d A$ and $\Phi \equiv \int_{A} q(t, x) d A$ be the total time traveled and total distance traveled, respectively, over a (discrete) time-space region $A$. By letting the symbol $A$ also denote its area, Edie's [7] average density, flow and speed in $A, k_{A}, q_{A}$ and $v_{A}$, respectively, are given by:

$$
k_{A} \equiv \Psi / A, \quad q_{A} \equiv \Phi / A, \quad v_{A} \equiv q_{A} / k_{A}
$$

These general definitions can be greatly simplified in our case because the spatiotemporal solution to our problem only involves 3 traffic states: capacity state $\mathrm{C}$ with (flow, density) $=(1 / 2,1 / 2)$, the empty or void state $\mathrm{O}$ with $(0,0)$ and the jam state $\mathrm{J}$ with $(0,1)$; see figure $\mathrm{xx}$. The total area that each state occupies in $A$ is indicated with a subscript, and they satisfy:

$$
A=A_{0}+A_{C}+A_{J}, \quad A_{J}=A_{0} \rightarrow A_{C}=A-2 A_{J}
$$

The total travel time $\Psi=\int_{A_{C}} \frac{1}{2} \cdot d A+\int_{A_{J}} 1 \cdot d A=$ $A_{C} / 2+A_{J}$, and the total distance traveled $\Phi=A_{C} / 2$. Using (9) we have:

$$
\Psi=A / 2, \quad \Phi=A / 2-A_{J}
$$

and therefore $A_{J}<A / 2$ is bounded. Also,

$$
k_{A}=1 / 2, \quad q_{A}=1 / 2-p, \quad v_{A}=1-2 p
$$

where $p=A_{J} / A$ correspondence to the probability that a time-space point in $\mathrm{A}$ is congested. It can be seen that the total time-space area in congestion, $A_{J}$, turns out to be the key measure of performance from which all others can be derived.

\section{F. Probability densities}

The exposition in this section is simplified by taking the continuum limit of the discrete problem analyzed so far, where the size of a cell and the duration of a time step both vanish. Here the symbols $L, A$ the denote dimensional variables in units of distance and area, respectively.

The main result here is that when the area $A=L^{2} / 4$ is of triangular shape as in Fig. 1, area $A_{J}$ corresponds to half the area under a Brownian excursion, which is known to have the Airy distribution for the unit length road segment $[1,9,17]$; let $g(a), 0 \leq a \leq 1$ be its PDF. Fig. 3 plots the PDF of the Airy distribution, $g(a), 0 \leq$ $a \leq 1$; notice that it is not a heavy-tail distribution. For arbitrary length $L$ the area under the excursion becomes $L^{-3 / 2} g\left(a L^{-3 / 2}\right)$ [17], and the PDF of $A_{J}$ is:

$$
f_{A_{J}}(a)=2 L^{-3 / 2} g\left(2 a L^{-3 / 2}\right)
$$

The distribution of all other traffic flow variables will be given by different scalings of the Airy distribution. It 


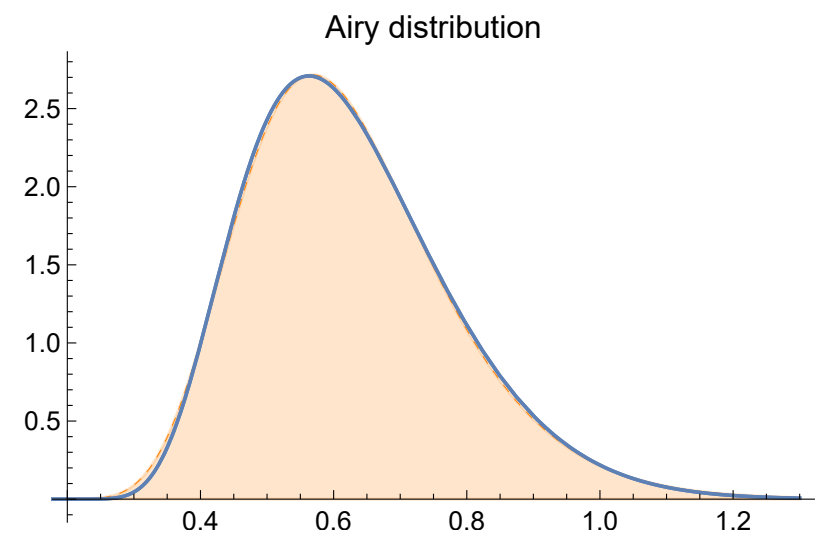

Figure 3. PDF of the Airy distribution, $g(a), 0 \leq a \leq 1$, in blue. Although it is analytical, there is no closed-form expression for this distribution, only a complicated infinite sum [17], which is not implemented by default in most libraries/packages (the first 24 terms in this sum were considered for making this figure). The shaded area corresponds to the area under the Inverse Gaussian Distribution with mean parameter 0.625416 and shape parameter 9.82985 , which can be seen to provide an accurate (and fast) approximation.

follows from (11) the PDF for these variables is given by

$$
\begin{aligned}
f_{p}(a) & =A f_{A_{J}}(a A)=g\left(a L^{1 / 2} / 2\right) L^{1 / 2} / 2 \\
f_{q_{A}}(a) & =f_{p}(1 / 2-a) \\
f_{v_{A}}(a) & =f_{p}((1-a) / 2) / 2
\end{aligned}
$$

which only depend upon $L^{1 / 2}$. It can be seen from (13a) that the Airy distribution can be interpreted as the likelihood that a particular point in $A=L^{2} / 4$ is congested on a segment of length $L=4$.

A Brownian excursion is a conditioned one dimensional Brownian motion over the time interval $(0, T)$ such that its path starts and ends at the origin and it is constrained to stay positive in between. It is important to note that the area under the excursion is also given by the area between a Brownian bridge and its minimum value in $(0, T)$. This second interpretation is precisely our context: (i) exactly at the critical density the number of empty and unoccupied cells are equal so that in our ran- dom walk $f(0)=f(T)$, which corresponds to a Brownian bridge, and (ii) from Fig. 1 it can be seen that (half) the total area under the random walk and its minimum value is $A_{J}$, since:

$$
A_{J}=\sum_{i=1}^{m} H_{i}
$$

where $m$ is the total number of elementary jams in the system. Unlike (7), the correlations among the $H_{i}$ 's plays a significant role here because the sum across all elementary jams in the system is limited by the size of the system itself.

\section{DISCUSSION}

This paper has shown additional evidence that traffic flow at the critical density is fractal in nature, with self-similarities at all scales. In the $(t, x)$-plane the basic fractal "generator" unit is a triangle similar to the fundamental diagram, containing 3 traffic states: voids, capacity and jams.

Starting with random initial conditions, we have shown that on long segments congestion clusters propagate for distances that follow a power law with exponent $1 / 2$. This indicates that the average propagation distance (or time) of a jam, and its variance, do not exist, and therefore jams of any size may be expected for no particular reason at the critical density.

Although we have used arguably the simplest traffic flow model, the results presented here should apply in general, as a consequence of our conjecture of universality of CA-184.

Although the case for Hurst exponents $H>0.5$ is strong for both cumulative counts and flow time series, it remains to be established that the fractional process approach is justified in the particular case analyzed in this paper.

\section{ACKNOWLEDGMENT}

This research was funded by NSF Awards \#1932451 and \#1826162.
[1] Agranov, T., Zilber, P., Smith, N.R., Admon, T., Roichman, Y., Meerson, B., 2020. Airy distribution: Experiment, large deviations, and additional statistics. Physical Review Research 2, 013174.

[2] Aschwanden, M.J., 2014. A macroscopic description of a generalized self-organized criticality system: Astrophysical applications. The Astrophysical Journal 782, 54.

[3] Bak, P., Tang, C., Wiesenfeld, K., 1987. Self-organized criticality: an explanation of $1 / \mathrm{f}$ noise. Phys. Rev. Lett 59,381 .
[4] Chand, S., Aouad, G., Dixit, V.V., 2017. Long-range dependence of traffic flow and speed of a motorway: Dynamics and correlation with historical incidents. Transportation Research Record 2616, 49-57.

[5] Daganzo, C.F., 2005. A variational formulation of kinematic wave theory: basic theory and complex boundary conditions. Transportation Research Part B 39, 187-196.

[6] Dănilă, B., Harko, T., Mocanu, G., 2015. Self-organized criticality in a two-dimensional cellular automaton model of a magnetic flux tube with background flow. Monthly 
Notices of the Royal Astronomical Society 453, 29822991.

[7] Edie, L.C., 1965. Discussion of traffic stream measurements and definitions, in: 2nd Int. Symp. on Transportation and Traffic Theory, Paris, France. p. 139???154.

[8] Halperin, B., Hohenberg, P., 1969. Scaling laws for dynamic critical phenomena. Physical Review 177, 952.

[9] Janson, S., 2007. Brownian excursion area, wright's constants in graph enumeration, and other brownian areas. Probability Surveys 4, 80-145.

[10] Kobayashi, Y., Takayasu, H., Havlin, S., Takayasu, M., 2021. Robust characterization of multidimensional scaling relations between size measures for business firms. Entropy 23, 168.

[11] Kostinski, S., Amir, A., 2016. An elementary derivation of first and last return times of $1 \mathrm{~d}$ random walks. American Journal of Physics 84, 57-60.

[12] Krause, S.M., Habel, L., Guhr, T., Schreckenberg, M., 2017. The importance of antipersistence for traffic jams. EPL (Europhysics Letters) 118, 38005.

[13] Laval, J., 2021. Power law behavior of elementary cellular automata. Preprints 2021060649. doi doi: 10.20944/preprints202106.0649.v1.

[14] Laval, J.A., Chilukuri, B.R., 2016. Symmetries in the kinematic wave model and a parameter-free representation of traffic flow. Transportation Research Part B: Methodological 89, 168 - 177.

[15] Laval, J.A., Leclercq, L., 2013. The Hamilton-Jacobi partial differential equation and the three representations of traffic flow. Transportation Research Part B 52, 17-30. doi doi:10.1016/j.trb.2013.02.008.

[16] Lighthill, M.J., Whitham, G.B., 1955. On kinematic waves ii. a theory of traffic flow on long crowded roads. Proceedings of the Royal Society of London. Series A. Mathematical and Physical Sciences 229, 317-345.

[17] Majumdar, S.N., Comtet, A., 2005. Airy distribution function: from the area under a brownian excursion to the maximal height of fluctuating interfaces. Journal of Statistical Physics 119, 777-826.

[18] Mori, T., Smith, T.E., Hsu, W.T., 2020. Common power laws for cities and spatial fractal structures. Proceedings of the National Academy of Sciences 117, 6469-6475.

[19] Nagel, K., 2021. Personal communication.

[20] Nagel, K., Paczuski, M., 1995. Emergent traffic jams. Physical Review E 51, 2909.

[21] Nagel, K., Rasmussen, S., Barrett, C.L., 1996. Network traffic as a self-organized critical phenomena. Technical Report. Los Alamos National Lab., NM (United States).

[22] Olmos, L.E., Çolak, S., Shafiei, S., Saberi, M., González, M.C., 2018. Macroscopic dynamics and the collapse of urban traffic. Proceedings of the National Academy of Sciences 115, 12654-12661.

[23] Paczuski, M., Nagel, K., 1996. Self-organized criticality and $1 / \mathrm{f}$ noise in traffic, in: Traffic and granular flow, World Scientific Singapore. p. 73.

[24] Richards, P.I., 1956. Shock waves on the highway. Operations research 4, 42-51.

[25] Rieser, M., Nagel, K., 2008. Network breakdown "at the edge of chaos" in multi-agent traffic simulations. The European Physical Journal B 63, 321-327.

[26] Schadschneider, A., 2021. Personal communication.

[27] Schroeder, M., 2009. Fractals, chaos, power laws: Minutes from an infinite paradise. Courier Corporation.
[28] Wolfram, S., 1984. Cellular automata as models of complexity. Nature 311, 419.

[29] Zaliapin, I., Kagan, Y.Y., Schoenberg, F.P., 2005. Approximating the distribution of pareto sums. Pure and Applied geophysics 162, 1187-1228.

[30] Zeng, G., Gao, J., Shekhtman, L., Guo, S., Lv, W., Wu, J., Liu, H., Levy, O., Li, D., Gao, Z., et al., 2020. Multiple metastable network states in urban traffic. Proceedings of the National Academy of Sciences 117, 17528-17534.

[31] Zeng, G., Li, D., Guo, S., Gao, L., Gao, Z., Stanley, H.E., Havlin, S., 2019. Switch between critical percolation modes in city traffic dynamics. Proceedings of the National Academy of Sciences 116, 23-28.

[32] Zhang, L., Zeng, G., Li, D., Huang, H.J., Stanley, H.E., Havlin, S., 2019. Scale-free resilience of real traffic jams. Proceedings of the National Academy of Sciences 116, 8673-8678.

[33] Zipf, G., 1949. Human behaviour and the principle of least effort. 